\title{
On the meaning and the epistemological relevance of the notion of a scientific phenomenon
}

\author{
Jochen Apel \\ Department of Philosophy, University of Heidelberg, \\ Schulgasse 6, Heidelberg, 69117, Germany, \\ e-mail: j.apel@uni-heidelberg.de
}

Post-print with some corrected typos. First published in Synthese (2011) 182, 23-38, DOI 10.1007/s11229-009-9620-y

\begin{abstract}
In this paper I offer an appraisal of James Bogen and James Woodward's distinction between data and phenomena which pursues two objectives. First, I aim to clarify the notion of a scientific phenomenon. Such a clarification is required because despite its intuitive plausibility it is not exactly clear how Bogen and Woodward's distinction has to be understood. I reject one common interpretation of the distinction, endorsed for example by James McAllister and Bruce Glymour, which identifies phenomena with patterns in data sets. Furthermore, I point out that other interpretations of Bogen and Woodward's distinction do not specify the relationship between phenomena and theories in a satisfying manner. In order to avoid this problem I propose a contextual understanding of scientific phenomena according to which phenomena are states of affairs which play specific roles in scientific practice and to which we adopt a special epistemic attitude. Second, I evaluate the epistemological significance of Bogen and Woodward's distinction with respect to the debate between scientific realists and constructive empiricists. Contrary to what Bogen and Woodward claim, I argue that the distinction does not provide a convincing argument against constructive empiricism.
\end{abstract}

Keywords: Data, Phenomena, Bogen, Woodward, Constructive empiricism, van Fraassen 


\section{Introduction}

In their seminal paper Saving the Phenomena James Bogen and James Woodward attack philosophical models of science according to which scientific theories predict and explain observable facts, which in turn provide evidence for or against those theories. They argue that a closer look at scientific practice reveals that one should better distinguish between data, which are the observed outcomes of measurements, and phenomena, which are explained and predicted by theories but have to be inferred from data. Bogen and Woodward take it to be a serious shortcoming of most philosophical accounts of science that they do not take this difference into account.

In this paper I scrutinize how exactly the distinction between data and phenomena has to be understood and what its philosophical impact is. So, the aim of my discussion is twofold. First, I try to provide some conceptual clarification. I seek to answer the question what exactly is meant when something is described as a phenomenon. I argue that several ways of understanding the notion, which are endorsed in the philosophical literature, are not appropriate to capture its meaning. In particular there seems to be no unambiguous interpretation of the distinction between data and phenomena. Therefore, I will make a proposal how the notion of a scientific phenomenon and Bogen and Woodward's distinction are understood best. En passant I will argue that in the light of this proposal an objection Bruce Glymour (2000) has raised against Bogen and Woodward turns out to be misguided.

Second, I want to evaluate Bogen and Woodward's claim that the nonconsideration of the data-phenomena-distinction is a serious shortcoming of traditional philosophical accounts of science. If this thesis is correct Bogen and Woodward's distinction should shed some new light on problems we encounter in philosophy of science. Of course, in a short paper like the one at hand I cannot generally assess whether it does so or not. Rather the second aim of the paper is more modest. I only try to show that Bogen and Woodward's thesis does not hold in one particular case. I argue that the data- phenomenadistinction is of no philosophical importance for the debate between scientific realists and constructive empiricists. Bogen and Woodward attempt to show that this central issue in contemporary philosophy of science is a prime example where their distinction displays its philosophical value by providing an argument against constructive empiricism. I will argue to the contrary: the data-phenomena-distinction does not force us to take any different point of view within this important epistemological debate. 
My paper's structure is as follows: In Sect. 2 I identify two conceptual features which are characteristic for the notion of a phenomenon in scientific parlance. Section $\underline{3}$ deals with the traditional empiricist view of scientific phenomena and James Bogen and James Woodward's critique of it which is based on their distinction between data and phenomena. In Sect. $\underline{4} \mathrm{I}$ argue that despite its intuitive plausibility it is not exactly clear how this distinction has to be understood. I reject one widespread interpretation of it which I call the pattern view of scientific phenomena. In Sect. $\underline{5}$ I point out that other interpretations of Bogen and Woodward's distinction also suffer from obscurities. In order to avoid these unclarities I propose a contextual understanding of scientific phenomena. Finally, in Sect. 6 I evaluate the philosophical significance of Bogen and Woodward's distinction with respect to van Fraassen's constructive empiricism.

\section{Conceptual features of the notion of a scientific phenomenon in scientific parlance}

The expression "phenomenon" is a common part of scientists' vocabulary. Consider for example the following two statements taken from a recent issue of Nature.

In the phenomenon known as the Aharonov-Bohm effect, magnetic forces seem to act on charged particles such as electrons - even though the particles do not cross any magnetic field lines. Is this evidence for electromagnetic forces that work in new and unsuspected ways? Tonomura and Nori (2008, p. 298)

The presence of a liquid-gas transition was noted to be very remarkable because there are few, if any, other experimentally known instances in localized spin systems. [...] No mechanism was known to account for this phenomenon, and our theory of magnetic monopoles fills this gap. Castelnovo et al. (2008, p. 44)

I take these statements to be typical examples for scientists' talk about phenomena. ${ }^{1}$ Therefore, it is possible to extract general characteristics of the notion from them: each statement points to a specific role, which phenomena play in scientific practice. Those roles can be stated as follows:

\footnotetext{
${ }^{1}$ That these statements really are such typical examples is of course an empirical hypothesis. But I think it is easy to persuade oneself from its correctness, e.g., by typing the word "phenomenon" into the online search of databases of scientific journals and reading through the hits.
} 
(a) Phenomena are potential evidence for scientific theories (first quotation) and

(b) phenomena are potential explananda of scientific theories (second quotation). ${ }^{2}$

These two roles provide the starting point for my further discussion. In the following two sections I examine two philosophical proposals for the explication of the notion of a scientific phenomenon. Those proposals spell out the notion by invoking epistemic criteria; that means they restrict its usage to entities about which we gain knowledge in specific ways. But as it will turn out, those proposals fail at specifying the notion in way that respects its yet identified characteristic features.

Of course, such a line of argumentation presupposes that the criticized proposals are descriptive approaches, i.e., that these proposals are intended to analyze the notion of a phenomenon as it is used by scientists. Obviously, if those proposals were rather meant as stipulative approaches, which use the expression "phenomenon" as a technical term with a meaning stipulated exclusively for a certain philosophical theory, my argumentation would be pointless. For this reason I will have to point out in the next section why the discussed proposals and in particular Bogen and Woodward's account of phenomena should be understood as attempts to illuminate what scientists mean when they describe something as a phenomenon.

\section{Traditional accounts of science and the distinction between data and phenomena}

The historical roots of the expression "phenomenon" as a part of the scientific vocabulary can be traced back to the ancient astronomical research program called 'saving the phenomena'. Here the observed motions of the celestial bodies where denoted as "phenomena" (cf. Mittelstrass 1961). Ancient astronomical theories should account for these motions without violating the principles of Greek natural philosophy. A generalization of this conception of phenomena, which I call the traditional view of scientific phenomena, can also be found in contemporary philosophy of science. The traditional view identifies phenomena with the observable structures and processes of the world. Such a view

\footnotetext{
${ }^{2}$ One might wonder why I added the qualification "potential" in (a) and (b), but the reason for this is simple. In many cases scientists encounter a phenomenon while possessing neither a theory to explain it nor one for which the phenomenon provides evidence, but are rather in search for such theories. Nevertheless we want to say that those scientists encountered a phenomenon.
} 
is typically held by empiricists like Pierre Duhem (1969) or Bas van Fraassen (1980). Both claim that it is the aim of science 'to save the phenomena', thereby explicitly echoing the name of the ancient research program. Saving the phenomena means for van Fraassen that scientific theories should make true statements about the observable structures and processes of the world, but we cannot and need not know whether what our theories say about the unobservable parts of the world is true or not. So, according to the traditional view the criterion for being a phenomenon is an epistemic one, namely that we can acquire knowledge about it by observation. Closely connected with the traditional view is a conception of science according to which science can be modeled as consisting out of two parts: an observational basis and a theoretical superstructure. The observational basis confirms theories and the theories in turn allow for explanation and prediction of observable phenomena.

James Bogen and James Woodward (1988) challenge this dyadic picture by introducing the distinction between data and phenomena into the philosophical discourse (cf. also Bogen and Woodward 1992, 2003; Woodward 1989, 2000). According to Bogen and Woodward the phenomena of science are typically unobservable. We rather have to infer them from reliable scientific data. Scientific theories, in turn, do not explain and predict observed data but inferred phenomena.

Bogen and Woodward characterize data and phenomena in the following ways: Data are perceivable and public accessible records of measurement outcomes. They are, as they put it, "idiosyncratic" (Bogen and Woodward 1988, p. 317), which means that they are bound to specific experimental contexts and typically do not occur outside of scientists' laboratories. Furthermore, data result from complex causal interactions in such a way that it is usually not possible to construct theories which explain or predict their occurrence. Phenomena, in turn, are not idiosyncratic. They rather are "stable and general features of the world" (Woodward 1989, p. 393), for which our theories should account. The very same phenomenon can often be detected via different experimental methods, while those methods yield different kinds of data. Bogen and Woodward criticize traditional accounts of science for leaving out these important differentiations and plead for a modification of traditional conceptions of science: the dyad of theory and observation should be replaced by a triad of data, phenomena and theories.

To exemplify the main idea let us briefly consider their notorious example for a phenomenon: the melting point of lead. Scientists do not determine the melt- 
ing point of lead by liquefying one sample of lead and observing an individual thermometer reading of the melting temperature. Instead they perform a series of measurements and even when the measuring device functions properly and systematic errors can be excluded the various individual thermometer readings (i.e., the data) in this measurement series will differ slightly from one another. In order to explain this one assumes that the measurement results were determined as well by the 'true melting point' as by numerous small and contingent 'errors', which cannot be adequately controlled and which often remain even unknown. If we can assure that those errors are independent from one another, have the same variance and are leading with equal probability to an increase as to a decrease of the measured value we are justified in claiming that the data are normally distributed and that the arithmetic mean is a good estimate for the 'true value'. The establishment of the phenomenon that the melting point of lead is, say, $327.46 \pm 0.04{ }^{\circ} \mathrm{C}$ therefore involves various levels of data analysis and inference. Consequently, the melting point of lead is nothing we observe at all; rather this fact has to be inferred from observed data mainly on the basis of statistical inference.

At the end of Sect. 2 I introduced a distinction between descriptive and stipulative approaches and pointed out that I take Bogen and Woodward as pursuing the former type of account. Having introduced Bogen and Woodward's distinction in this section I am now in a position to make clear why I think that the framework they provide should be understood in this manner. First, the ways Bogen and Woodward characterize phenomena seem to be not only coherent with scientists' usage of the notion, but even explicitly motivated by the fact that what scientists regard as phenomena often differs from how philosophers conceptualize scientific phenomena in their accounts. Second, Bogen and Woodward do not explicitly attempt to give a definition of the term "phenomenon", which is something one would suspect if the expression was used as a stipulated technical term. Third, the descriptive understanding fits general developments in philosophy of science. Nowadays descriptive approaches are regarded as more successful than stipulative ones. Just think of the notorious debate about the nature of theories. There we try to find out what theories are particularly by investigating which kinds of objects scientists describe as such. We regard it for example as a shortcoming of the syntactic view that it does not apply to things to which scientists' routinely refer as a theory, e.g., Darwinian evolutionary theory. If we accept that such descriptive strategies are the most successful means of philosophical investigation the principle of charity also suggests to take Bogen and Woodward as proponents of such a strategy. 
For these three reasons I take it that the data-phenomena-distinction is really meant to illuminate the concept of phenomena (and the concept of data as well) as we find it in scientific parlance.

\section{The pattern view of scientific phenomena and the detection of extrasolar planets}

If the traditional identification of phenomena with observable structures and processes is misguided in the way Bogen and Woodward claim, how can we then specify what phenomena are? In the following I discuss one particular interpretation of the data-phenomena-distinction which one frequently finds in the philosophical literature. I will refer to this interpretation as the pattern view of scientific phenomena. This view is motivated on the one hand by the just mentioned melting point example and on the other hand by statements like the following from Woodward's paper Data and Phenomena:

The problem of detecting a phenomenon is the problem of detecting a signal in this sea of noise, of identifying a relatively stable and invariant pattern of some simplicity and generality with recurrent features - a pattern which is not just an artifact of the particular detection techniques we employ or the local environment in which we operate. Woodward (1989, p. 396)

Authors like James McAllister (1997) or Bruce Glymour (2000) take statements like this to assert that phenomena are or correspond to particular patterns in data sets. These patterns can typically be represented graphically, for example by drawing a smooth curve through a set of data points. Patterns correspond to phenomena in the sense that it is assumed that those patterns can be found not only in the examined samples but also in the whole population. For example it is assumed that not only the experimentally analyzed lead samples exhibit the pattern that lead melts at $327.46 \pm 0.04{ }^{\circ} \mathrm{C}$ but every sample of lead will do so under appropriate circumstances. According to this view phenomena are what the statistician calls population statistics and the criterion for being a phenomenon which the pattern view invokes is therefore again an epistemic one, namely that knowledge about phenomena is gained exclusively by statistical interpretations of observable data. ${ }^{3}$

\footnotetext{
${ }^{3}$ At this point the question arises whether Bogen and Woodward themselves also endorse the pattern view. As I said, some authors suggest this, but to me this does not seem to be correct. This can be seen for example from the fact that Bogen and Woodward also regard weak neutral currents as a phenomenon (cf. for example Bogen and Woodward 1988, p. 315). This obviously does not fit the pattern view. But I do not want to discuss exegetic questions here. For me it is enough that there are authors that endorse the pattern view interpretation to make it expedient to examine how well this suggestion works.
} 
As aforementioned, one of the authors which endorse the pattern view interpretation of Bogen and Woodward's distinction is Bruce Glymour. This in turn leads him to the conclusion that the whole distinction between data and phenomena is unnecessary and philosophically uninteresting. His objection against Bogen and Woodward is straightforward and runs as follows (cf. Glymour $\underline{2000}$, pp. 33, 34): If phenomena are population statistics, then Bogen and Woodward simply give a new name to a well-known distinction from statistics. Instead of talking about sample and population statistics they just use the other words namely "data" and "phenomena". Therefore, the data-phenomenadistinction does not provide any new insights for philosophy of science and is for this reason nothing more than a superfluous and at the worst misleading terminological reform.

In the remainder of this section I argue that the pattern view interpretation of Bogen and Woodward is misguided. Phenomena cannot be identified with patterns in data sets. I will elaborate on this by considering the first discovery of extrasolar planets. My thesis is that the existence of extrasolar planets should also be regarded as a scientific phenomenon although this phenomenon does not correspond to a pattern in a data set. En passant these considerations lead to a rejection of Glymour's objection because his critique presupposes the pattern view interpretation.

Extrasolar planets or, for short, exoplanets are planets outside of our solar system. For a long time it was an open question whether there are planets orbiting other stars than our sun or not. Astronomers were interested in this question for different reason. One reason is of course that the correct answer could give us a hint whether there possibly exists life in other parts of the universe. A further important reason was that the detection of extrasolar planets would provide (and as a matter of fact did provide) strong evidence for certain astronomical theories about the formation of stars and planets. According to those theories the formation of planets is a typical by-product of the formation of stars and therefore there should be numerous exoplanets out there (cf. for example Casoli and Encrenaz 2007, pp. 91-116).

If we now ask how the distinction between data and phenomena can be applied to the first detection of an exoplanet we have to reconsider the detection method by which the astronomers Michael Mayor and Didier Queloz discovered this planet. This method is called velocimetry and it depends on the measurement of the radial velocity of the star 51 Pegasi (cf. Casoli and Encrenaz 2007, pp. 22-30). Radial velocity is the component of the velocity of a moving ob- 
ject whose vector points directly to the observer (or directly away from her). ${ }^{4}$ If one measures the temporal development of the radial velocity of 51 Pegasi one finds that it shows a periodical change over time. From this change astronomers infer the existence of an exoplanet which causes this change by its gravitational interaction with the star. If one takes a look at following figure from Mayor and Queloz's original paper one seems to be confronted with a paradigmatic case for the pattern view of phenomena (Fig. 1).

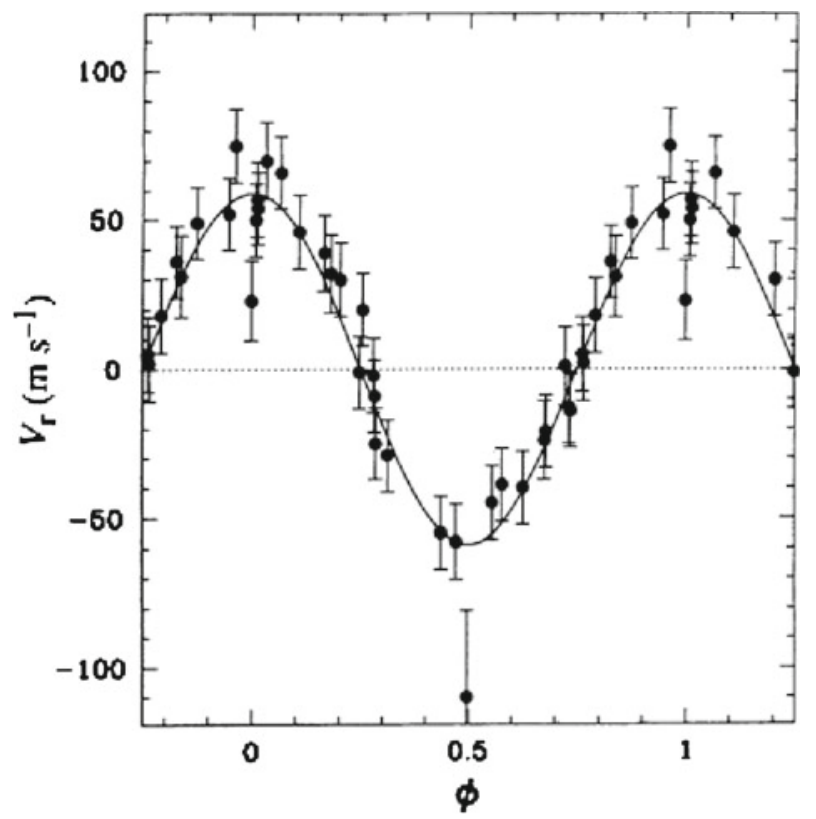

Fig. 1 Radial velocity change of 51 Pegasi (Mayor and Queloz 1995, p. 357)

The figure displays a distribution of data points fitted by a sinus curve. This curve represents the periodical change of 51 Pegasi's radial velocity and according to the pattern view one should say that the only phenomenon Mayor and Queloz detected was this periodical change because it is the only thing which corresponds to a pattern in a data set. Against this I argue that it was another phenomenon Mayor and Queloz ultimately detected, namely the fact that 51 Pegasi is orbited by a planetary companion. Taking the existence of this exoplanet as a phenomenon can draw on the following reasons:

\footnotetext{
${ }^{4}$ To be more precise: One actually measures the Doppler-shift of the light emitted from the star and infers from this to the radial velocity change. But because it does not make a difference to my argument I skip this intermediate step for the sake of simplicity.
} 
First, this fact plays the roles which are characteristic for phenomena: it provides evidence for astronomical theories about planetary formation and on the other hand it is this fact that can be explained by those theories as soon as they become accepted. ${ }^{5}$ Second, the change of 51 Pegasi's radial velocity in contrast to the existence of the exoplanet does not provide strong evidence for astronomers' theories about planetary formation, because this change could also be brought about by causes different from the gravitational influence of the extrasolar planet. For example the very same pattern could have been caused by a pulsation of the star or by so-called spot rotation. ${ }^{6}$ Scientists are interested in evidence which provides good reasons for hypotheses and the pattern alone cannot provide such, because in order to possess good reasons one has to rule out the mentioned rival hypotheses. But in doing so one does nothing else than establishing the exoplanet hypothesis. ${ }^{7}$

Third, treating the existence of extrasolar planets as a phenomenon fits very well with our everyday usage of the expression "phenomenon". Here phenomena are things that are surprising, noteworthy, sometimes exceptional and for which we would like to have an explanation-and in the end it was the existence of extrasolar planets what astronomers were really curious about and not the radial velocity pattern.

Fourth - this point complements the third - for astronomers nothing hinged on the question which of the different available methods was used to establish the exoplanet's existence. Other detection methods would have been as appropriate as the one they actually applied, e.g., astrometrical or photometrical methods. But those methods would have brought about totally different kinds of data and consequently totally different kinds of patterns in data sets.

I want to clarify, however, that I do not deny that the periodical change of 51 Pegasi's radial velocity is a scientific phenomenon; I only argue that the pattern view is not universally valid. As the phenomenon of extrasolar planets reveals to identify a phenomenon we often have infer from patterns in data sets

\footnotetext{
${ }^{5}$ As the following quote from a standard textbook on astrophysics illustrates also astronomers refer routinely to extrasolar planets or the fact that those exist as a phenomenon: "A proper understanding of phenomena like black holes, quasars and extrasolar planets requires that we understand all physics that underlies all of astrophysics." (Duric 2003, p. i).

${ }^{6}$ These are not mere theoretical possibilities invented by philosophers, who argue for some kind of underdetermination thesis, but real-life possibilities seriously considered by astronomers (cf. Mayor and Queloz 1995, pp. 357-358).

${ }^{7}$ This consideration points to certain insufficiencies of so-called positive relevance conceptions of evidence. Arguments that scientific evidence is indeed something over and above positive relevance can be found in Achinstein (2001, pp. 5-10).
} 
like the periodical change of the radial velocity to the causes of these patterns like the orbiting of the exoplanet, which brings about this change. Therefore, I conclude that the notion of a scientific phenomenon cannot be adequately explicated by the pattern view. Furthermore it results from these considerations that Glymour's way of arguing that the data-phenomena-distinction is superfluous is not convincing because his critique relied solely on the misguided pattern view.

It should be noted, however, that this rebuttal of Glymour's objection is built on the assumption that Bogen and Woodward really subscribe to a descriptive approach as I described it in the previous sections, because only in this case the deficiency of the pattern view interpretation inevitably makes Glymour's critique lapse. On the contrary, if Bogen and Woodward were instead pursuing a stipulative approach Glymour might rightly call the relevance of their distinction into question. This gives a further, indirect reason to understand Bogen and Woodward as pursuing the former type of approach.

\section{Theories and claims about phenomena: a contextual approach}

What we found out in the preceding sections is that analyses which tie the notion of a scientific phenomenon to our ways of epistemic access to the relevant entities are not convincing. As a matter of fact science often deals with phenomena which are neither directly observable nor statistically inferred patterns in data sets. In this last step I discuss a question which results from the fact that none of the proposed epistemic criteria proved as universally valid to specify what phenomena are. This question is how phenomena and claims about phenomena are related to theories. Which of the claims scientists make are descriptions of phenomena and which are theoretical accounts for those phenomena?

In a first approximation the relation between theories and phenomena is something like this: According to the syntactic view presenting a theory is stating a few, maybe quite abstract axioms which form a deductive system. By feeding some additional information into this system for example certain boundary conditions we can derive theorems from these axioms. At least some of those theorems are then the parts of the theory which correspond to phenomena. On the rival semantic view, in turn, presenting a theory is presenting a family of (set theoretic) models. Those models or at least substructures of them correspond to phenomena. Thus, on both accounts phenomena correspond to substructures of the whole theoretical structure. But which substructures exactly 
correspond to phenomena? While discussing this question I will adopt the following manner of speaking: I will describe substructures which correspond to phenomena as claims about phenomena and the rest of the theoretical structure as theoretical claims. By adopting this terminology I do not want to imply that my considerations are only valid if one follows the syntactic view. Rather I think that they hold independently from which conception of theories one prefers. My mode of speaking is a mere terminological convention which I adopt because it corresponds to Bogen and Woodward's parlance.

I will argue that there is no clear-cut, context-independent criterion by which claims about phenomena and theoretical claims can be distinguished. Rather claims about phenomena are a subset of the theoretical claims to which we adopt special epistemic attitudes. This thesis constitutes a difference to the yet discussed conceptions of scientific phenomena. According to those there is a clear-cut distinction between claims about phenomena and theoretical claims. A proponent of the traditional view simply draws the distinction this way: The claim "Coffee cups fall to the ground when they drop from the table." is a claim about a phenomenon, because we can observe accordant states of affairs. In turn all claims in which unobservable entities figure have to be considered as theoretical, for example the claim "There is a gravitational force specified by Newton's law of gravitation which makes the coffee cup fall". ${ }^{8}$ An analogous distinction can be drawn for the pattern view with respect to claims about patterns in data sets.

But if one does not want to stick to one of these problematic views it is no longer clear which claims of science belong to mere descriptions of phenomena and which belong to theory. Nevertheless, Bogen and Woodward also suggest that there is a clear-cut distinction between both, but they draw it on different grounds. According to them, only theoretical claims have the potential to provide scientific explanations of claims about phenomena while claims about phenomena only figure as explananda but not as explanantia. Data, phenomena and theories can therefore be distinguished by the kinds of explanatory relations between them:

- Claims about data do not explain and are not explained. They are neither explananda nor explanantia.

\footnotetext{
${ }^{8}$ This does not presuppose a distinction between observational and theoretical vocabulary. The only relevant point is whether the sentence refers to observable or unobservable entities (cf. van Fraassen 1980, p. 15).
} 
- Claims about phenomena are explained by theories but do not explain claims about data. They are explananda but no explanantia.

- Theoretical claims explain claims about phenomena and can be explained by other theoretical claims. They are explanantia but can also be explananda.

The basic idea can again be illustrated by the melting point example. The melting point of lead can be explained by physical theory, be it thermodynamics or a theory about the atomic bindings in solid state bodies. But these theories and also the phenomenon of the melting point of lead itself cannot be invoked to explain the individual data points. They do not explain why we measured one time $326.8^{\circ} \mathrm{C}$ and the other time $327.1^{\circ} \mathrm{C}$.

But is the proposed distinctive criterion that claims about phenomena, in contrast to theoretical claims, are not used to provide scientific explanations really feasible? Consider for example the phenomenon of extrasolar planets and an accordant claim about this phenomenon:

P1: The star 51 Pegasi is orbited by a planetary companion.

This phenomenon is inferred as a cause of the periodical change of the radial velocity and therefore it can of course be invoked to explain this periodical change. For example envisage a student of astronomy who has basis knowledge in physics and astrophysics but does not know why the radial velocity change occurs. It is obvious that you can explain the change to him by referring to the phenomenon that there is an exoplanet orbiting the star. Consequently claims about phenomena can very well provide explanations for other phenomena and are in this respect on a par with theoretical claims. ${ }^{9}$

Furthermore, it is not even clear whether claims about phenomena really do not explain data or at least aspects of them. In order to discuss this, consider the following sentences:

P2: Lead melts (under such and such circumstances) at $327.46 \pm 0.04^{\circ} \mathrm{C}$.

$\mathrm{X}$ : In this experiment (consisting in a series of measurements of melting temperatures of lead samples with a certain measuring instrument) the arithmetic mean of the melting temperatures was $326.43^{\circ} \mathrm{C}$.

\footnotetext{
${ }^{9}$ Note that this is not an idiosyncrasy of the exoplanet case but holds true for many other phenomena, for example for phenomena of atomic and subatomic physics. E.g., the phenomenon of weak neutral currents can be invoked in the very same manner to explain the central characteristics of bubble chamber trails.
} 
$\mathrm{X}$ is a description of a certain aspect of experimental data because $\mathrm{X}$ is explicitly bound to an experimental context. Being bound to specific experimental contexts is a characteristic of data and not of phenomena. But contrary to Bogen and Woodward, I think that can P2 provide an explanation of X.

This is so because explanations typically involve a contextually determined contrast class and there are at least some contrast-classes with respect to which P2 is explanatory. E.g., if one asks for an explanation of the fact that we found in this particular experiment a mean value of $326.43^{\circ} \mathrm{C}$ and not of, say, $370^{\circ} \mathrm{C}$ one could respond to this question by stating P 2 and adding that the experimental situation to which $\mathrm{X}$ refers approximately meets the circumstances specified in P2. Maybe one might want to call into question whether this is really an explanation, but such doubts can be allayed. Admittedly the given explanation is not a very deep one, but nevertheless it is explanatory at least in a minimal sense. Explanations are answers to why questions, they are requests for certain pieces of information. And stating that every sample of lead has the dispositional property of melting at $327.46 \pm 0.04^{\circ} \mathrm{C}$ under appropriate circumstances can provide the requested information in an appropriate context. Just think of other materials like paraffin (a certain type of wax) which do not have a single melting point. A comparison with this case makes plausible that the average melting behavior of certain lead samples, i.e., experimental data, can very well be explained by referring to the stable melting point of lead. If lead would not have this property we would have measured different data which most likely would not have been normally distributed. In this sense there is a pattern of counterfactual dependency between the statistical properties of the considered data set and property of having a melting point of $327.46 \pm 0.04^{\circ} \mathrm{C}$. According to Woodward's own account on explanation, explanatory relations are grounded exactly in such patterns of counterfactual dependency (cf. Woodward and Hitchcock 2003, p. 11). These considerations provides further reason to assume that claims about phenomena can as well as theoretical claims figure as explanantia.

Therefore, I conclude that the suggestion of a clear-cut, context-independent distinction between claims about phenomena and theoretical claims is untenable. Rather theories seem to be nothing else than putative descriptions of phenomena. Our theory of atomic bindings, for example, describes phenomena of atomic binding and states relations between the different phenomena it describes. Why do we then sometimes refer to a certain state of affairs as a phenomenon while we are in other cases more reluctant to do so? There certainly are cases in which we are more likely to say things like that our description of 
a certain states of affairs is 'just' a theoretical hypothesis and not a claim about a phenomenon.

This indicates that we regard certain states of affairs as phenomena if we adopt a special epistemic attitude towards these states of affairs. Denoting a state of affairs as a phenomenon expresses that (additional to the ascription of two roles identified in Sect. 2) one assumes to have good reasons for accepting a corresponding claim which describes this state of affairs. Phenomena are state of affairs which we accept as given in a certain context. And this is the case if the claim figures as highly probable given our experimental data and accepted background knowledge. If we talk about the phenomenon of exoplanets or the phenomenon of weak neutral currents we accept the claims that there are exoplanets orbiting stars and that there are weak neutral currents in certain particle interactions.

Consequently there is no difference in principle between theoretical claims and claims about phenomena; rather there is just a contextual determined difference. To be more specific I claim that one should distinguish between strongly accepted claims of science, which are claims about phenomena, and claims which are less strong accepted. These claims one might call "theoretical hypothesis". Claims about phenomena and theoretical hypothesis together make the whole set of theoretical claims. A claim can be regarded as theoretical hypothesis in one and as a claim about a phenomenon in another context, for example when a change in background theory or the acquisition of new experimental data raises (or lowers) our acceptance of the claim.

That our epistemic attitudes determine whether something is a phenomenon squares well with the two roles of phenomena which were identified in Sect. 2: Something we accept in a strong manner is of course more appropriate to provide evidence for further hypotheses than something we are not so sure of. An analogous point holds true for the explanandum role: we only ask for an explanation if we have good reasons for accepting the explanandum-sentence. Thus, the notion of a phenomenon is a contextual concept which can be characterized in the following manner:

(i) States of affairs can be regarded as phenomena only if they play certain roles in science: They have to be potential evidence for and potential explananda of further scientific claims.

(ii) Claims about such states of affairs are judged as claims about phenomena only if there are sufficiently good reasons to accept those claims. 
There are no further restrictions on states affairs in order to qualify as phenomena. In particular there is no restriction regarding our way of epistemic access to the relevant states of affairs. There are various ways by which we can acquire knowledge about phenomena. Some phenomena can be directly observed like the phenomenon that the heaven is blue or that lead melts if you heat it strongly, some correspond to patterns in data sets like the melting point of lead or the periodical change of the radial velocity of 51 Pegasi and some we infer as causes of such patterns like the phenomenon of extrasolar planets or the existence of weak neutral currents.

\section{Data, phenomena and constructive empiricism}

In the last section of this paper I want to shift the focus from the analysis of the meaning of the expression "phenomenon" as it is used in scientific discourse to the question whether we can gain something for the philosophical metadiscourse about science from the distinction between data and phenomena. Here I will focus on epistemological issues related to the scientific realism debate and argue that at least in this particular field we do not gain new insights from the distinction. Bogen and Woodward by contrast claim that van Fraassen's constructive empiricism, arguably the most prominent rival account to scientific realism in contemporary philosophy of science, is unable to accommodate the distinction between data and phenomena into its theoretical framework (cf. Bogen and Woodward 1988, pp. 349-352; Woodward 1989, pp. 450-452).

At the heart of constructive empiricism lies the notion of empirical adequacy. The aim of science according to constructive empiricism is to find empirically adequate theories, i.e., theories which make true statements about the observable. But in contrast to the scientific realist the constructive empiricist holds the view that we should be agnostic with regard to the truth-value of scientific claims about the unobservable parts of the world. Bogen and Woodward object that the consideration of the distinction between data and phenomena renders van Fraassen's construal of empirical adequacy implausible. They write:

Empirical adequacy, as we understand it, means that a theory must "save" or "be adequate to" the phenomena, which for the most part are not observed, rather than the data which are observed. By contrast, van Fraassen requires that theories save or be adequate to what can be observed. This is tantamount to requiring that a theory must save the data-that an acceptable theory of molecular structure, in Nagel's example [i.e., the melting point example; J.A.], must fit the observed scatter of thermometer readings, rather than the true melting point of lead which is inferred from these readings. We have argued 
at length that this is an unreasonable requirement to impose on any theory. It seems unlikely that van Fraassen could accept our notion of empirical adequacy without abandoning many of his most central claims. Bogen and Woodward (1988, p. 351)

To my knowledge van Fraassen nowhere reacts to this challenge, but prima facie there seem to be two options he could take in order to maintain constructive empiricism. The first option would be to argue that the data-phenomenadistinction shows that the realm of the unobservable is larger than commonly expected. Despite what one intuitively thought Bogen and Woodward have shown that also phenomena like the melting point of lead are in fact unobservable and the constructive empiricist has to bite the bullet: She also has to be agnostic with regard to the truth-value of claims about those phenomena just as well as in the case of any claim in which unobservable entities figure.

But taking this option cannot be satisfying for van Fraassen because he claims that his position gives an as adequate account of scientific practice as scientific realism. That means in particular that its determination of the aim of science should be coherent with actual scientific practice. Indeed his position is advocated by van Fraassen as the "equilibrium point" between epistemic modesty and adequacy to science (Monton and van Fraassen 2003, p. 407; cf. also BergHildebrand and Suhm 2006). But if it is the case that science is mostly concerned with unobservable phenomena and that there are virtually no observable phenomena at all then this casts serious doubts on van Fraassen's claim that it is the aim of science to find empirically adequate theories, if empirical adequate theories are understood as theories which make true statements about data. Hence, if van Fraassen really were committed to taking this option the data-phenomena-distinction would indeed show that constructive empiricism does not meet the criterion of adequacy to science, which van Fraassen himself imposes.

But, as I will argue in the remainder of this paper, there also is a second option according to which van Fraassen is in not committed to such a narrow understanding of empirical adequacy but should better construe this notion in a way such that it includes patterns in data sets.

The defense of van Fraassen's position goes like this: Van Fraassen readily concedes that nearly every claim about a phenomenon involves more than pure observation but also some kind of inference. This can be seen from the fact that constructive empiricism contains a commitment to the truth about the observable and not just about the observed. This step already involves some ob- 
servation-transcending inference and van Fraassen is willing to take the epistemic risk involved this ampliative inference in order to meet the adequacy to science criterion. Analogously to these observed-observable inferences he can further concede that the adequacy to science criterion also requires the acceptance of inferences by which we move from data to patterns in data sets. Nevertheless he can still maintain that those inferences involve a lesser degree of epistemic risk than inferences to phenomena involving unobservable entities. Everything one needs to establish the melting point of lead are observable data about melting temperatures of observable lead samples, assumptions about the absence of systematic error and statistical methods of data analysis. ${ }^{10}$ But those assumptions and mathematical inferences, so van Fraassen might argue, do not increase epistemic risk in the same way as theoretical inferences to the existence of unobservable entities like electrons do, because they do not involve any specific physical theory to license the observation transcending step; in particular they do not involve any new ontological commitments (Cf. van Fraassen and his coauthors in Ladyman et al. 1997, p. 317, for a similar line of argument). Contrary to Bogen and Woodward's objection, such an argumentation enables him to stretch the notion of empirical adequacy in a way that it covers patterns in data sets while his position is still epistemically more modest than scientific realism. Furthermore, it seems as if van Fraassen already endorses such a broad understanding of empirical adequacy anyway when he writes:

The whole point of having theoretical models is that they should fit the phenomena, that is, fit the models of the data. van Fraassen (1985, p. 271)

Here he refers to phenomena explicitly as "models of data", a terminus introduced by Patrick Suppes (1962). But those are nothing else than what was called patterns in data sets in this paper. Hence, the practice of saving the phenomena (in van Fraassen's sense) might very well include patterns in data sets and at the same time exclude states of affairs in which unobservable entities figure.

It is worth noting that, contrary to what Bogen and Woodward claim, such a broad understanding of empirical adequacy is still in accordance with its definition as truth in regard to the observable. That phenomena which correspond to patterns in data sets are often not observed but inferred does by no means entail that those phenomena are unobservable. Rather, under appropriate cir-

\footnotetext{
${ }^{10}$ Remember that van Fraassen has no quarrels with the claim that also our observational vocabulary is theory-infected (cf. van Fraassen 1980, p. 14).
} 
cumstances, i.e. in the absence of all confounding factors, we would observe, e.g., lead samples melting exactly at their melting temperature. This case is perfectly analogous to the fact that dinosaurs are observable because we would observe dinosaurs under appropriate observation conditions regardless of whether we are de facto able to realize those conditions.

In summary, nothing about the distinction between data and phenomena debars the empiricist from claiming that we should have different epistemic commitments with regard to different types of phenomena depending on whether the accordant states of affairs involve observable or unobservable entities. There may be other reasons why there are no epistemological significant differences between both kinds of phenomena but such reasons would be independent of the data-phenomena-distinction.

\section{Concluding remarks}

My appraisal of Bogen and Woodward's distinction between data and phenomena yielded two main results. The first result is a clarification of the notion of a scientific phenomenon. I argued that ways of understanding the notion which specified phenomena via the way we gain knowledge about them were not able to account for the characteristic features of the notion in scientific parlance. It is neither an essential property of phenomena to be observable, as the traditional view claims, nor is it that phenomena solely correspond to unobservable patterns statistically inferred from data sets, as the pattern view has it. A broader reading of Bogen and Woodward's distinction which also allows for the inclusion of, e.g., the phenomenon of extrasolar planets seemed to provide a more promising attempt to capture the notion's meaning but seemed at the same time to be connected with an unclear distinction between claims about phenomena and theoretical claims. In order to deal with this difficulty I provided an analysis which does not specify the notion by connecting it to a special way of epistemic access to phenomena but rather by the roles of scientific phenomena in scientific practice and by our epistemic attitudes towards claims about phenomena.

The second result is an evaluation of the epistemological significance of Bogen and Woodward's distinction for the debate between scientific realists and constructive empiricists. The upshot of this investigation is that the distinction does not provide any convincing arguments for this debate. 


\section{Acknowledgments}

I am deeply indebted to Daniela Bailer-Jones for initiating my work on scientific phenomena and I thank various people, who gave valuable comments on earlier drafts of this paper, especially Monika Dullstein, Pavel Radchenko, Stephan Hartmann and the participants of conference "Data-PhenomenaTheories" held in Heidelberg in 2008. My research is financially supported by the "Emmy-Noether-Programm der Deutschen Forschungsgemeinschaft".

\section{References}

Achinstein, P. (2001). The book of evidence. Oxford: Oxford University Press..

Berg-Hildebrand, A., \& Suhm, C. (2006). The hardships of an empiricist. In A. Berg-Hildebrand \&

C. Suhm (Eds.), Bas C. van Fraassen-The fortunes of empiricism (pp. 5767). Frankfurt/Main: Ontos..

Bogen, J., \& Woodward, J. (1988). Saving the phenomena. The Philosophical Review, 97, 303-352.

Bogen, J., \& Woodward, J. (1992). Observations, theories and the evolution of the human spirit. Philosophy of Science, 59, 590-611.

Bogen, J., \& Woodward, J. (2003). Evading the IRS. Poznan Studies in the Philosophy of Science and the Humanities, 20, 223-245.

Casoli, F., \& Encrenaz, T. (2007). The new worlds: Extrasolar planets. Berlin: Springer.

Castelnovo, C., Moessner, R., \& Sondhi, S.L. (2008). Magnetic monopoles in spin ice. Nature, 451, 42-45.

Duhem, P. (1969). To save the phenomena-An essay on the idea of physical theory from Plato to Galileo. Chicago: University of Chicago Press.

Duric, N. (2003). Advanced astrophysics. Cambridge: Cambridge University Press.

Glymour, B. (2000). Data and phenomena: A distinction reconsidered. Erkenntnis, 52, 29-37.

Ladyman, J., Douven, I., Horsten, L., \& van Fraassen, B. C. (1997). A defence of van Fraassen's critique of abductive inference: Reply to Psillos. The Philosophical Quarterly, 47, 305-321. 
Mayor, M., \& Queloz, D. (1995). A Jupiter-mass companion to a solar-type star. Nature, 378, 355-359.

McAllister, J. W. (1997). Phenomena and patterns in data sets. Erkenntnis, 47, $217-228$.

Mittelstrass, J. (1961). Die Rettung der Phänomene-Ursprung und Geschichte eines antiken Forschungsprogramms. Berlin: Walter de Gruyter \& Co.

Monton, B., \& van Fraassen, B. (2003). Constructive empiricism and modal nominalism. British Journal for Philosophy of Science, 54, 405-422.

Suppes, P. (1962). Models of data. In E. Nagel, P. Suppes, \& A. Tarski (Eds.), Logic, methodology, and philosophy of science-Proceedings of the 1960 international congress (pp. 252-261). Stanford: Stanford University Press.

Tonomura, A., \& Nori, F. (2008). Disturbance without the force. Nature, 452, 298-299.

van Fraassen, B. (1980). The scientific image. Oxford: Clarendon Press.

van Fraassen, B. (1985). Empiricsm in the philosophy of science. In P. M. Churchland \& C. A. Hooker (Eds.), Images of science (pp. 245-368). Chicago: University of Chicago Press.

Woodward, J. (1989). Data and phenomena. Synthese, 79, 393-472.

Woodward, J. (2000). Data, phenomena, and reliability. Philosophy of Science, 67, S163-S179. Woodward, J., \& Hitchcock, C. (2003). Explanatory generalizations, Part I: A counterfactual account. Nous, 37, 1-24. 